International Journal of Biomedical Engineering and Science (IJBES), Vol. 3, No. 4, October 2016

\title{
STUDYING THE IMPACT OF ALCOHOL CONSUMPTION AND ITS CULTURAL UNDERSTANDING ON EEG USING EMBEDDING DIMENSION IN YOUNG HEALTHY ADULTS FROM SHILLONG, MEGHALAYA
}

\author{
Arundhuti Das ${ }^{1}$, Shrimanta Ramchiary ${ }^{2}$, Sudip Paul ${ }^{2}$, Tapas Sinha ${ }^{2}$ and Dinesh \\ Bhatia $^{2} *$ \\ ${ }^{1}$ Department of Anthropology, Utkal University, Bhubaneswar-751004, Odisha \\ ${ }^{2}$ Department of Biomedical Engineering, North Eastern Hill University, Shillong- \\ 793022, Meghalaya
}

\begin{abstract}
Cultural understanding and environment of upbringing plays a pivotal role in deciding right and wrong that influences our perception and decision-making. Electroencephalogram (EEG) is being employed since past several years to understand cognitive functioning of human brain. Here, we employed EEG to determine impact in cognitive functioning due to cultural upbringing. The objective was to detect if there is any influence of difference in cultural understanding related to alcohol use, on EEG obtained from alcohol consumers and non-consumers. The present study was done between Indo-Aryan and Tibeto Burman linguistic groups from Shillong Meghalaya. Embedding dimension was used to characterize EEG. The embedding dimension from the frontal and occipital regions of the brain of both alcohol consumers and non-consumers in Tibeto Burmans and Indo Aryans were determined via a MATLAB program. The results show that the embedding dimension profiles of alcohol consumers and non-consumers are quite different. For the control (non consumers) both in case of Tibeto Burman and Indo Aryans the value of the embedding dimension was higher in occipital regions as compared to the alcohol consumers. This result implies the loss of coherence and neuronal connectivity for alcohol consumers. However, this effect is more prominent in the case of Indo Aryans.
\end{abstract}

\section{KEYWORDS}

Embedding dimension, EEG, alcohol consumption, cultural difference .

\section{INTRODUCTION}

Human brain is composed of neuronal network that is arranged spatially and the electroencephalogram (EEG) captures these spatial electrical activities in the brain [1]. Various electrophysiological methods are used to evaluate brain dysfunction; electroencephalogram (EEG) is one such method. Consumption of alcohol has shown to cause various kinds of malfunctions in the brain. It also reduces the total grey matter volume [2]. The impact of alcohol consumption in the brain dysfunction has been measured using EEG [3-4]. In a study done by Ehlers [5] they demonstrated the impact of low drinking and moderate drinking on EEG bands. They observed that low doses of alcohol tend to increase slow alpha activity or lowering of alpha peak frequency, while moderate doses show increase in slow alpha and theta bands. 
Alcoholism as a trait is multifactorial; it's causal is contributed by genetics, culture, and environment. In families with drinking history and in families with no history of alcoholism, EEG is different. Individuals with positive family history of alcohol consumption show greater increase in alpha waves than the individuals with no family history [6]. Thus, the impact of the environment one is introduced to while growing up and the family's understanding of alcohol consumption plays a significant role in dictating brain's response to alcohol consumption as can be detected using EEG. The electroencephalogram has also been used to understand how culture get embrained, it has shown the impact and difference of culture can be measured using EEG and thus a proof that cultural difference does make a difference in neural networking which has been measured using EEG [7]. There have been studies between alcohol consumers of different ethnic groups. A study done on family with positive Hispanic history, individuals' show decreased fast alpha while non-Hispanics showed an increase in the same band.

There are various methods used to characterize and analyze EEG for example Lyapunov exponents, autocorrection function and correlation dimension. Correlation dimension has been successfully used in detecting relative changes in EEG obtained from Schizophrenic patients [8], Alzheimer's patient [9], Alcohol Users [10]. Embedding dimension, which is derived using correlation dimension, helps construct the arrangement of neural signals spatially recorded using EEG. It has been used to explore the brain mechanism in Schizophrenia [8] and epilepsy [11].

Cultural acceptance of alcohol or its traditional use in ritual influences ones consumption pattern. The cultural environment is one in which an individual is brought up predisposed to perceive an incident and undergo decision-making. Amongst many tribes in North Eastern region of India, tobacco and alcohol consumption are culturally learned. In the present study, participants were from the Indo Aryan and Tibeto Burman linguistic group, the individuals from the former linguistic group do not consume alcohol as a cultural custom unlike the Tibeto- Burman group, where alcohol consumption is part of their cultural custom. They have traditional forms of alcohol for example rice beer, which is used in different traditional and religious practices as an important integrant. Thus, the perception related to alcohol consumption is considered as a food habit rather than an addictive substance, unlike in Indo- Aryan culture where it is culturally considered as a restricted beverage.

\section{METHODOLOGY}

Subject acquisition: Permission to conduct the study was sought from the Institutional Ethical Committee for Human Participants (IECHSP), North-Eastern Hill University, Shillong, Meghalaya (Ref. No. IECHSP/2015/20). The study was conducted amongst young adults between the age group 20-30 (mean 21 \pm 2 ), out of which $75 \%$ were male and $25 \%$ female. The participants were from two different linguistic groups, Indo-Aryans and Tibeto-Burmans. This study was conducted with 20 individuals. The data was collected after informed consent was obtained from the participant. The sample collection was done from Shillong, Meghalaya region only.

Study design: Data collection was done in two stages: (A) Psychological test and qualitative open ended questions regarding socio-psychological parameters followed by (B) EEG data was collected to address the neurobiology aspect.

\subsection{Stage 1}

Demographic information such as socioeconomic status, educational status of the subject, educational status of family, age, gender, ethnic group, subgroup, and linguistic group was collected. Anthropological questions were asked related to alcohol consumption in order to understand the cultural acceptance of alcohol consumption or not, for example if the participants have any traditional liquor, which is consumed during cultural or religious events. The questions were asked to evaluate the acceptance of alcohol consumption (or not) in participant's family, 
International Journal of Biomedical Engineering and Science (IJBES), Vol. 3, No. 4, October 2016

they were also inquired whether any member of their family consumes alcohol. These questions would help us determine the social priming of the individual towards alcohol usage.

\subsection{Stage 2}

\section{EEG equipment and EEG recording montages}

EEG signals were collected using the recording system with suitable bio-amplifier (Power lab $26 \mathrm{~T}$ from M/s. AD Instruments, Australia). In this study, we used two channels at a time for continuous 10 minutes recording with selected $0.1 \mathrm{~Hz}$ Low Pass filter, $70 \mathrm{~Hz}$ High Pass filter and $50 \mathrm{~Hz}$ Notch filter for removing artifacts from the raw EEG signals. In our study, the sampling rate was 256 [12]. The EEG instrument itself did the digitalization. A bipolar electrode montage system was used for recording the human brain EEG signal. Electrodes were implanted bilaterally in the skull over the frontal and occipital region of the brain. A reference electrode was placed over the forehead. The frequency band of $0.5-4 \mathrm{~Hz}$ was expressed as delta, $4-8 \mathrm{~Hz}$ as theta, 8-13 $\mathrm{Hz}$ as alpha, $14-25 \mathrm{~Hz}$ as beta and $25-45 \mathrm{~Hz}$ as gamma. They were asked to refrain from alcohol consumption for 24 hours before the EEG data was collected.

\subsection{Post Data Collection}

MATLAB was used to analyze the EEG recording. Statistical analysis was done using MS EXCEL, SPSS version 16 statistical software. Case control analysis between the alcohol consumer and non- consumer group for the EEG parameters were done using One- way ANOVA analysis in SPSS.

\subsection{Correlation Dimensions}

The correlation dimension (denoted by $v$ ) is a measure of the dimensionality of the space occupied by a set of random points, often referred to as a type of fractal dimension [13]. For example, if we have a set of random points on the real number line between 0 and 1 , the correlation dimension will be $v=1$, while if they are distributed on say, a triangle embedded in three-dimensional space (or m-dimensional space), the correlation dimension will be $v=2$. This is what we would intuitively expect from a measure of dimension. The real utility of the correlation dimension is in determining the (possibly fractional) dimensions of fractal objects. Considering the time series: $\left\{V\left(t_{i}\right), V\left(t_{i}+\tau\right), \ldots V\left(t_{i}+(n-1) \tau\right)\right\}$, the integral correlation coefficient is obtained (Babloyantz et al., 1986). Here one starts with a reference point $V_{i}$ and its distance to the rest of the points is computed. Then one computes how many points lie within a given distance $r$ from $V$. Repeating this process for all values, we get $C(r)$, the integral correlation coefficient of the attractor. For small $r, C(r) \sim r^{v}$ where $v_{\text {is }}$ the correlation dimension and is given by the slope of the plot of $\log (C(r))$ versus $\log (r)$. The slope was computed directly in the Matlab program for various embedding dimension. With the help of cf tool in MATLAB we evaluated the embedding dimension for both the cases.

\section{RESULTS}

The result obtained from correlation dimension analysis of EEG from occipital region of brain in case of Tibeto Burman participants are shown in Figure 1. The results of alcohol consumers and non-consumers are shown in two colour-coded waves, the red colour indicates the controls or non-consumers. The blue colour represents alcohol consumers. These results indicate that there is 
International Journal of Biomedical Engineering and Science (IJBES), Vol. 3, No. 4, October 2016

a phase shift between the alcohol consumers and non-consumers. The embedding dimension in alcohol non-consumers is 1.25 , where as the embedding dimension in alcohol consumers is 1.04.

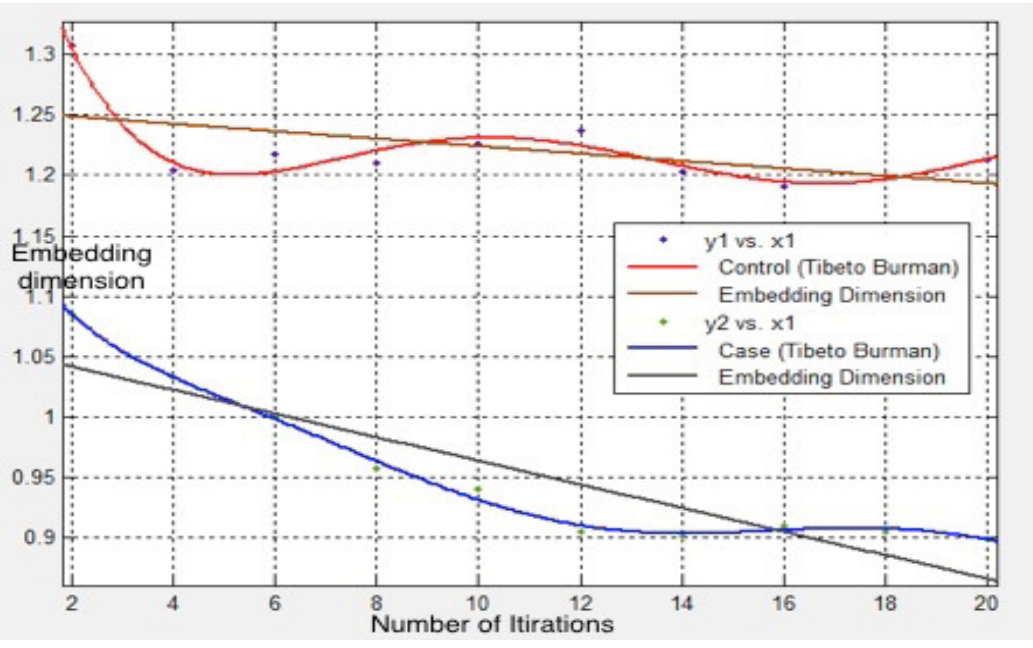

Figure 1: showing correlation dimension of occipital region in Tibeto Burman alcohol consumers and non consumers

Figure 2 shows the correlation dimension obtained from occipital region in alcohol consumers and non-consumers in case of the Indo Aryan participants. The red line indicates correlation dimension in controls (alcohol non consumers) and the blue line indicates correlation dimension in cases (alcohol consumers). The embedding dimension in alcohol consumers is 1.24 while that in controls is 0.94 .

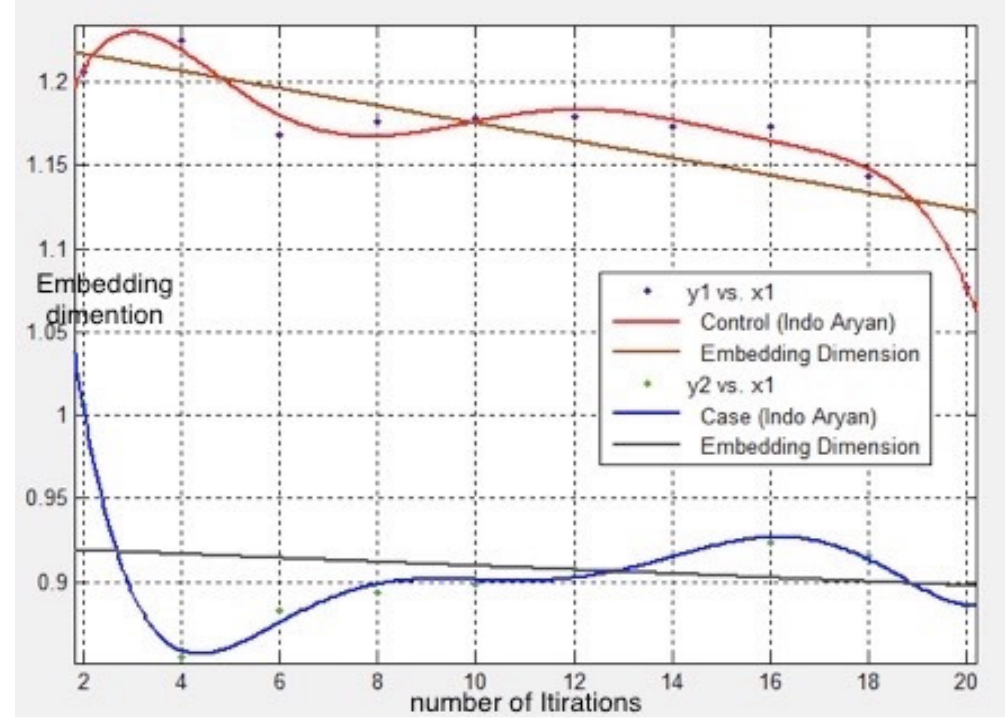

Figure 2: showing correlation dimension of occipital region in Indo aryan alcohol consumers and non consumers 
International Journal of Biomedical Engineering and Science (IJBES), Vol. 3, No. 4, October 2016

\section{DISCUSSION}

The present study shows significant differences between the EEG obtained from alcohol consumers and non-consumers. EEG was obtained from frontal and occipital regions of the brain. The participants were from the Tibeto-Burman and Indo-Aryan linguistic groups. They were further divided into two groups namely the alcohol consumers and non-consumers. The nonconsumers are an absolute control group, where the participants had never consumed alcohol. In the two groups studied, the Tibeto-Burman represents a culture where alcohol consumption as traditional drink is common practice. There are traditional form of alcoholic beverage, which is consumed at functions, rituals and gatherings. These traditional alcoholic drinks are also offered to local deity during religious functions and ceremonies. Thus, the tradition and concept of alcohol consumption in their culture is not attached with taboos and guilt. However in the case of Indo-Aryans, cultural norms represent a total rejection to alcohol consumption. Thus, its use is associated with guilt and taboos. In the present study major difference in the embedding dimension is seen between the consumers and non-consumers in individuals from both the group (Tibeto-Burman and Indo- Aryan) however, the impact is higher on the Indo-Aryans as compared to Tibeto-Burman. The EEG data was characterized using correlation dimension. The embedded dimension measures have been used to characterize EEG obtained due to induced stroke in rats [14]. Changes in the EEG from frontal region of human brain, due to alcohol consumption has been observed in previous studies [15]. This results shows that constant use of alcohol reduces coherence between neurons both in occipital region. Interaction between neurons is also reduced due to alcohol consumption. The impact was higher in the occipital of the brain of Indo-Aryans. In a study, done to see the effect of anesthesia on the frontal and the occipital lobe shows that the occipital lobe shows loss of spatial coherence in alpha oscillation [16]. The Frontal region of the brain is associated with modulation of emotional activity, while the occipital region of the brain is visual procession and perception. Impairment in visual processing is a significant disability induced due to alcohol use and abuse [17]. Studies have also shown brain shrinkage (reduction in grey matter volume) in alcoholics [2]. The present study has shown reduction in neural connectivity due to alcohol consumption, which is suggestive of diminishing efficiency of the region due to alcohol consumption. The effect being more prominent in individuals where culturally consumption of alcohol is forbidden. There have been studies in the past where EEG is used to measure how culture gets embrained [7]. Previous studies have shown that the impact of alcohol consumption is different between families where there is history of alcohol consumption compared to those where there is no history [6]. The present study also indicated in same direction, along with the fact that cultural understanding of alcohol consumption also influences EEG between consumers and non-consumers.

\section{CONCLUSION}

The study is in line with previous studies done by the same group [18] which also indicated a significant difference in embedding dimension between the alcohol consumers and non consumers in both frontal and occipital regions of the brain, the effect was stronger in occipital. As EEG captures the electrical activity of neurons in the spatial organization, embedding dimension characterized the EEG using dimensionality of the space occupied by the points. Reduced dimensionality indicates reduced interconnections. Further studies of this kind need to be carried out across various cultures to find the impact of cultural understanding and alcoholism on brain functioning, as was pointed out by the study.

\section{ACKNOWLEDGMENTS}

We would particularly like to thank all participants who took part in this study. We are thankful to UGC- Dr. D. S Kothari Postdoctoral Fellowship for their support 
International Journal of Biomedical Engineering and Science (IJBES), Vol. 3, No. 4, October 2016

\section{REFERENCES}

[1] E. Niedermeyer, F.L.D.Silva , Electroencephalography-Basic principles, clinical applications, and related fields. Baltimore: Lippincott Williams \& Wilkins, (1993)

[2] C. A. Paul, R. Au, L. Fredman, J. M. Massaro, S. Seshadri, C. DeCarli, and P. A. Wolf, "Association of Alcohol Consumption with Brain Volume in the Framingham Study," Archives of neurology., vol.65, no. 10, (2008),pp. 1363-1367.

[3] C. Acker, "Neuropsychological deficits in alcoholics: the relative contributions of gender and drinking history," British Journal of Addiction.,vol. 81, (1986), pp. 395-403.

[4] H.F. Moselhy, G. Georgiou, A. Kahn, "Frontal lobe changes in alcoholism: a review of the literature," Alcohol Alcohol., Vol. 36, (2001), pp357-368.

[5] C.L. Ehlers, E. Phillips, T.L. Wall , K. Wilhelmsen , M.A. Schuckit, "EEG alpha and level of response to alcohol in Hispanic- and non-Hispanic-American young adults with a family history of alcoholism,” J Stud Alcohol. , Vol.65, (2004), pp.301-308,.[PubMed: 15222586].

[6] H.L. Cohen, B. Porjesz, H. Begleiter, " The effects of ethanol on EEG activity in males at risk for alcoholism," Electroencephalogr Clin Neurophysiol. , Vol.86 (1993a) pp.368-376.. [PubMed: 7686471].

[7] Y.Yuan, Y Li and D. P Mandic, " Comparison analysis of embedding dimension between normal and epileptic EEG time series," The jounal of Physiologicl sciences advance publication., Vol.4, (2004)

[8] J. Jeong, D. J Kim, J.H. Chae, S.Y Kim, H.J Ko, I. H Paik, “ Nonlinear analysis of the EEG of schizophrenics with optimal embedding dimension," Medical Engineering and Physics., Vol.20, (1998), pp. 669-676,.

[9] J.Jeong, S.Y.Kim, S.H Han, “ Non- linear dynamical analysis of the EEG in Alzheimer's disease with optimal embedding dimension," Electroncephalography and Clinical Neurophysiology., Vol.106. No.3, (1998), pp.220-228.

[10] D.J. Kim, J. Jeong, K. S. Kim, J.H. Chae, S.H. Jin, K .J. Ahn, H. Myrick, S.J. Yoon, H.R. Kim and S. Y. Kim, "Complexity Changes of the EEG Induced by Alcohol Cue Exposure in Alcoholics and Social Drinkers," Alcoholism: Clinical and Experimental Research., Vol. 27, No. 12, (2003), pp.1955-1961.

[11] Y. Yuan, Y. Li and D.P. Mandic, “Comparison Analysis of Embedding Dimension between Normal and Epileptic EEG time series," The Journal of Physiological Sciences Advance.,vol.58, no.4, (2008), pp. 239-47.

[12] S. Paul, P. Bhattacharya, A.K. Pandey, N. Sharma, J.P. Tiwari and R Patnaik, " A Strategic Application of Fast Fourier Transform as a Novel Tool to Evaluate the Extent of Neuronal Insult in Rat Model of Focal Cerebral Ischemia,” Bangladesh Journal of Medical Physics., Vol. 5,No. 1, (2013), pp. 29-36,

[13] A.Babloyantz and A. Destexhe, "Low-Dimensional chaos in an instance of epilepsy," Proceedngs of the national academy of sciences., Vol.83, (1986) pp. 3513-3517.

[14] S.Paul, T.K.Sinha and R Patnaik, "EEG time series data analysis in focal cerebral ischemic rat model," International journal of Biomedical Engineering and Science., Vol. 2, No. 1,2015.

[15] M.Rangaswamy ,B. Porjesz , D.B. Chorlian, K. Wang, K.A Jones , S.Kuperman ,J. Rohrbaugh , S.J. O'Connor , L.O. Bauer , T. Reich, H. Begleiter , “ Resting EEG in offspring of male alcoholics: beta frequencies," Int J Psychophysiol. , Vol. 51, (2004), pp.239-251.

[16] P. L. Purdona, E. T. Piercea , E. A. Mukamelc, M. J. Preraua , J. L. Walsha , K. F.K. Wonga , A. F. S-Gomeza , P. G. Harrella , A. L. Sampsona, A. Cimensera, S. Chinga, N. J. Kopelle, C. T. Stoeckela, K. Habeebf , R. Merhara , and E. N. Browna, "Electroencephalogram signatures of loss and recovery of consciousness from propofol," Proc Natl Acad Sci., Vol. 110, No. 12, pp.E114251,2013Carlen, and D.A.Wilkinson, " Alcoholic brain damage and reversible deficits," Acta Psychiatrica Scandinavica.,vol. 62 (Suppl. 286), (1980) pp.103-118.

[17] A.V. Samokhvalov, S.Popova, R. Room, M. Ramonas and J. Rehm, "Disability Associated With Alcohol Abuse and Dependence," Alcoholism: Clinical and Experimental Research.,Vol.34, no.11, (2010) pp. 1871-1978.

[18] A.Das, S. Ramchairy, S. Paul, T. Sinha, D.Bhatia and P.K Das, "Understanding the impact of alcohol consumption on the EEG using embedding dimension in young healthy adults from Shillong, Meghalaya," (Manuscript submitted) 
International Journal of Biomedical Engineering and Science (IJBES), Vol. 3, No. 4, October 2016

\section{Authors}

Dr Arundhuti Das is a Dr D.S Kothari Postdoctoral fellow in the Department of Anthropology, Utkal University, Bhubaneswar, Odisha. Her research interest lies in the field of psychiatric genetics. She is working on different ethnic groups from India studying the genetic background behind traits like addiction that is perceived to have strong cultural predisposition in the respective environmental setting of the population. She did her Ph.D in Anthropology with specialization in anthropological genetics, Masters degree in Human Genetics and Bachelors degree in Biotechnology. She has won international scholarship twice from Balassi Institute, Budapest, Hungary for 10 months and 6 months respectively for partial $\mathrm{PhD}$ and research stay. She has presented her research work in Greece, Spain and USA.

Shrimanta S Ramchiary is a final year student in the Department of Biomedical Engineering, North Eastern Hill University. His field of interest lies in the field of Clinical engineering (pratical applications), neural systems engineering, imaging \& image processing, BioMems \& telemedicine.

Dr. Sudip Paul is currently an Assistant Professor in the Department of Biomedical Engineering, School of Technology, North-Eastern Hill University (NEHU), Shillong. He completed his B.Tech degree in Biomedical Engineering from West Bengal University of Technology, 2007; M.Tech degree in Biomedical Engineering from Banaras Hindu University, 2009 and also he completed his Ph.D degree from Indian Institute of Technology (Banaras Hindu University), Varanasi with specialization in

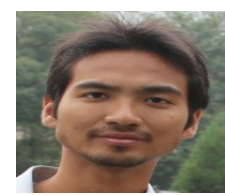
Electrophysiology and brain signal analysis in 2016. He has also presented his research accomplishments in USA, Greece, France and South Africa. He has many credentials in his credit out of which his First Prize in Sushruta Innovation Award 2011 sponsored by Department of Science and Technology, Govt. of India and also he also organized the 29th Annual meeting of the Society for Neurochemistry, India in 2015.

Dr. Tapas Sinha is currently working on Neural Network Based Pacemaker (DBT sanctioned project). He pursued his Ph.D in Physics from North Eastern Hill University, M.S in Physics and Biophysics from University of Cincinnati, USA. M.Sc is Physics from Benaras Hindu University (BHU). His field of research interest are Non Linear Dynamics, BioPhysics, Neural Networks.

Dr. Dinesh Bhatia pursued his $\mathrm{PhD}$ in Biomechanics and Rehabilitation Engineering from MNNIT, Allahabad, India in 2010 with Bachelor's (2002) and Master's degree (2004) in Biomedical Engineering from Mumbai University. He completed his MBA from IMT Ghaziabad in 2007. He is currently working as Associate Professor and Head, Department of Biomedical Engineering, North Eastern Hill University (NEHU), Shillong, Meghalaya, India since August 2013. Previously, he was employed as an Assistant Professor (Sr. Grade) at the Department of Biomedical Engineering,

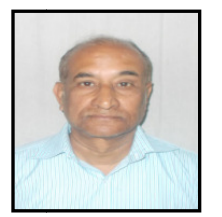
Deenbandhu Chhotu Ram University of Science and Technology, Murthal (Sonepat), Haryana (State Government University), India from July 2006 to July 2013. He was selected for the "Young Scientist Award (BOYSCAST)" in (2011-12) by Government of India to pursue research in osteoarthritis (OA) for one year at Adaptive Neural Systems Laboratory, Biomedical Engineering Department, Florida International University, Miami, Florida, USA. He is also the recipient of "INAE fellowship award" in 2011 by Indian National Academy of Engineering. He was selected as one of the twelve young Biomedical scientists by the ICMR, Govt. of India in 2015 to pursue research fellowship for 6 months in the field of sensory prosthetics at University of Glasgow, Scotland, UK. He has several research papers in reputed journals, conference, seminars and symposia with teaching and research experience of more than twelve years. He is invited panel member of many professional bodies, editorial boards, committees, societies and forums. He is currently having several funded projects from Government of India on physically challenged, disabled and paralyzed persons. He authored a book titled "Medical Informatics" published (2015) by PHI and "Stroke Rehabilitation" (2016) by ABS publishers. His research focuses on understanding muscle mechanics, joint kinematics and dynamics involved in performing locomotion and routine tasks and undermining it effects during an injury or disease. His interest areas are medical instrumentation, medical informatics, signal and image processing, biomechanics and rehabilitation engineering 